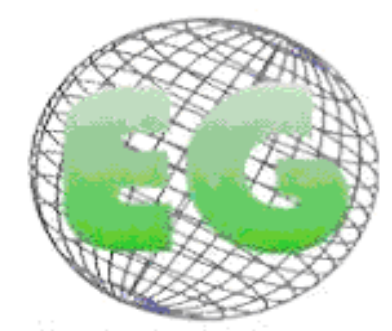

ISSN 1695-6141

$\mathrm{N}^{\circ} 24$

www.um.es/egloball

\title{
REVISIONES
}

\section{Enfermería basada en la evidencia y gestión del cuidado}

\author{
Evidence-based nursing and care management
}

${ }^{*}$ Rodríguez Campo, VA. **Paravic Klijn, TM.

*Magíster en Enfermería y Profesora de Biología. **Doctora en Enfermería . Profesora Titular Departamento de Enfermería. Universidad de Concepción. Chile.

Palabras clave: Enfermería basada en la Evidencia; Gestión del cuidado; Enfermería.

Keywords: Evidence based nursing, Management of care , Nursing..

\section{RESUMEN}

El presente trabajo tiene por objetivo describir la importancia de la Enfermería basada en la Evidencia como una estrategia relevante para el desarrollo con calidad de la Gestión del Cuidado, una de las funciones entregada en forma legal a la enfermera en año 1997, al ser incorporado en el Código Sanitario Chileno. Se revisaron los textos y documentos legales chilenos respecto al tema y artículos en diversas bases de datos, PubMed, SCielo, Cuiden Citación, Ciberindex. Describe las diversas etapas de la Enfermería basada en la Evidencia, así como las diversas funciones que se derivan de la Gestión del Cuidado y la importancia de la formación de los futuros enfermeras/os con esta estrategia de aprendizaje para entregar el mejor cuidado hacia los usuarios.

\section{ABSTRACT}

The aim of the study is to describe the importance of evidence-based nursing as an important strategy for an optimal development of the Management of Care, which is one of the nurses' tasks that was legally embodied when it was incorporated into the Chilean Health Code in 1997. The legal Chilean texts and documents were reviewed with regard to the topic and in articles in several databases - PubMed, Scielo, Cuiden Citacion, Ciberindex. It describes the several stages of evidence-based nursing as well as the several tasks that derive from the Management of Care and how important it is to implement this learning strategy to develop future nurses capable of offering their patients the best health care possible.

\section{INTRODUCCIÓN}

Los servicios profesionales de la enfermera comprenden la Gestión del Cuidado en lo relativo a promoción, manutención y restauración de la salud, la prevención de enfermedades o lesiones, y la ejecución de acciones derivadas del diagnóstico y tratamiento 
médico y el deber de velar por la mejor administración de los recursos de asistencia para el paciente. $^{(1)}$

La Gestión del Cuidado de enfermería será entendida como el ejercicio profesional de la enfermera sustentada en su disciplina: la ciencia del cuidar. Esta se define "como la aplicación de un juicio profesional en la planificación, organización, motivación y control de la provisión de cuidados, oportunos, seguros, integrales, que aseguren la continuidad de la atención y se sustenten en las políticas y lineamientos estratégicos de la institución". Por lo tanto, su fin último es ofrecer a los usuarios los mejores resultados posibles en la práctica diaria, acorde con la información científica disponible que haya demostrado su capacidad para cambiar de forma favorable el curso clínico de la enfermedad y que considere la mejor administración de los recursos, los menores inconvenientes y costos para el usuario y para la sociedad en su conjunto. ${ }^{(2)}$

Para alcanzar todo esto, una de las mejores estrategias, tal vez la mejor; es la EBE (Enfermería basada en la evidencia) que es definida como "proceso por el cual las enfermeras toman decisiones clínicas usando las mejores pruebas disponibles sustentadas en la investigación, su experiencia clínica y las preferencias del paciente, en el contexto de los recursos disponibles" (3) También se define como "la aplicación consciente, explícita y juiciosa de la mejor evidencia científica disponible relativa al conocimiento enfermero para tomar decisiones sobre el cuidado de los pacientes, teniendo en cuenta sus preferencias y valores, e incorporando la pericia profesional en esta toma de decisiones". (4) La EBE es un proceso en el que los problemas reales y potenciales que afectan la salud de los usuarios se presentan como preguntas, cuya respuesta se busca y evalúa sistemáticamente a partir de los resultados de la investigación más reciente, y que sirve de base para la toma de decisiones. Separar la investigación de la realidad clínica, es asumir el riesgo de dar una atención inadecuada, del mismo modo, si no se revisan las evidencias, los nuevos descubrimientos no son incorporados en los procesos y se ejecutan actividades de enfermería que pueden generar un perjuicio hacia el cliente. ${ }^{(5)}$

El gestionar el cuidado en forma oportuna, segura y satisfactoria lleva implícito el imperativo ético de responder frente a la sociedad con la mejor evidencia disponible para tomar las mejores decisiones sobre el cuidado de las personas.

Se revisaron los textos y documentos legales chilenos respecto al tema y artículos en diversas bases de datos, PubMed, SCielo, Cuiden Citación, Ciberindex.

\section{ENFERMERÍA BASADA EN LA EVIDENCIA}

El proceso de la EBE consta de cinco etapas o fases integradas dentro de un proceso dinámico y continuo que surge de la interacción paciente-enfermera que permite finalmente lograr la evidencia ${ }^{(3-6)}$ Considera el método científico como la mejor herramienta de que disponemos para conocer la realidad y expresarla de forma inteligible y sintética y reconoce la investigación científica como la actividad capaz de generar conocimiento válido y relevante para la moderna práctica profesional. Por lo tanto, se pone de manifiesto la contribución de la EBE a aumentar el cuerpo de conocimientos propio, lo que garantiza el desarrollo de la profesión enfermera.

La primera etapa se constituye por la formulación del problema o pregunta que se desea resolver, esta pregunta o problema de investigación surge de la práctica clínica diaria, de la gestión, de la docencia, que intentan responder a la incertidumbre o problemas cuestionados. ${ }^{(3,4,7)}$ 
La segunda etapa consiste en la búsqueda bibliográfica que brinde la mejor respuesta a la pregunta o problema planteado, para ello existen distintas fuentes de información, primarias o secundarias, que ayudan en la búsqueda de la mejor evidencia, como bases de datos, recursos bibliográficos electrónicos, catálogos, libros, publicaciones periódicas, Internet etc. $^{(8)}$ Es importante destacar, que la búsqueda de evidencia científica, se debe realizar de manera analítica, determinando la calidad de información contenida en las diversas fuentes antes mencionadas, a las cuales el profesional de enfermería acuda para mejorar su labor clínica y de docencia. ${ }^{(3,4)}$.

La tercera etapa consiste en evaluar la validez y utilidad de los hallazgos, se realiza una evaluación crítica de la evidencia encontrada y se clasifican las fuentes de información en los diferentes niveles y sus subclasificaciones. Cabe mencionar que la Agency for Healthcare Research and Quality tiene el valor de incluir los estudios de tipo cualitativo en esta etapa. ${ }^{(9)}$ La lectura crítica se conceptualiza como la capacidad consciente de adoptar una postura frente a lo expresado en artículos de investigación, se apoya en grados variables de conocimiento, reflexión y elaboración. Requiere tiempo para pensar, buscar, revisar, valorar y juzgar a partir de mínimos conocimientos en documentación, metodología de la investigación, estadística y epidemiología. Además, conocimiento sobre la práctica habitual y sus fundamentos como disciplina. ${ }^{(10)}$

En la cuarta etapa se realiza la implementación en la práctica de las evidencias científicas encontradas, teniendo como objetivo principal, mejorar el cuidado hacia el usuario, familia y comunidad. En la EBE es importante la opinión del usuario ${ }^{(5)}$ y en el acto de cuidar se debe tener en consideración las preferencias de la o las personas a quienes va dirigido. ${ }^{(3)} \mathrm{Al}$ momento de la implementación aparecen una serie de dificultades por lo que es indispensable desarrollar estrategias, identificar posibles barreras y conocer cuáles son las causas más frecuentes de fracaso para hacerlas frente. ${ }^{(3 ; 4 ; 6)}$

La última etapa del proceso de la EBE consiste en evaluar las consecuencias de la aplicación de la intervención elegida. Para la práctica de enfermería es importante, pues determina la retroalimentación entre investigación y clínica. Evaluar la repercusión de los cambios introducidos en la práctica, identificar si éstos han sido o no efectivos. La situación ideal es que la evaluación se lleve a cabo analizando resultados sensibles a las intervenciones de enfermería, con la finalidad de estimar los beneficios, daños y costes de las intervenciones. ${ }^{(4)}$

La EBE viene de la concepción de que el cuidado que brindamos está determinado por una investigación sólida y no por tradición o preferencia clínica, no cambia en base a un solo estudio, es la acumulación de los resultados de varios estudios, generalmente usando diversos diseños los que brindan evidencia suficiente para crear un cambio ${ }^{(11)}$. Amescua en González, ${ }^{(12)}$ plantea que "la EBE nos propone un cambio de modelo de una Enfermería basada en la obediencia (inscrita en el modelo biomédico) a una Enfermería basada en la (con)ciencia (modelo de cuidados)". El investigador es quien genera el conocimiento, pero es la comunidad profesional la que tiene que hacerlo práctico utilizando los resultados de la investigación.

Modelos de práctica en donde las enfermeras son anuladas, tienen efectos perniciosos en términos de morbimortalidad frente a modelos que fomentan la competencia, la toma de decisiones independiente y el trabajo en equipo. ${ }^{(13)}$

Sin embargo, a pesar de estudios realizados en diversos países sobre los beneficios de la EBE, surgen barreras que impiden implementar la EBE en la enfermería clínica, entre los cuales se mencionan insuficiente tiempo de las enfermeras para leer las investigaciones; 
organizaciones que no entregan las estructuras necesarias para aplicar las evidencias, dificultad de las enfermeras para comprender las estadísticas, sensación de aislamiento entre enfermeras para discutir los hallazgos y la falta de colaboración de los médicos. ${ }^{(14)} \mathrm{El}$ idioma en el cual se escriben las principales investigaciones, es otra limitante mencionada ${ }^{(15)}$ También se ha referido una escasa motivación en el estudio, bajas posibilidades de desarrollo profesional y falta de reconocimiento del trabajo de la enfermera ${ }^{(16)}$ Hay que agregar a ello, la falta de fuerza que ha tenido Enfermería para defender e implementar en los campos clínicos sus propios descubrimientos provenientes de las múltiples investigaciones realizadas, muchas de ellas con un gran esfuerzo, y cuyos resultados solo se han quedado en las tesis o revistas.

\section{GESTIÓN DEL CUIDADO y ENFERMERÍA BASADA EN LA EVIDENCIA (EBE)}

A partir del año 1997 los servicios profesionales de enfermería fueron reconocidos en el código sanitario chileno y se confiere a la profesión de enfermería tres grandes funciones: la gestión del cuidado, ejecutar acciones derivadas del diagnóstico y tratamiento médico y el deber de velar por la mejor administración de recursos de asistencia para el paciente. Este reconocimiento significa que el cuidado es el aporte específico de la enfermera en la atención de salud y, además, un ámbito determinado de esta atención en salud ha sido reservado para las enfermeras, con el objeto de otorgar certeza jurídica respecto a su campo de acción, de esta manera se garantiza el derecho a la protección de la salud del usuario. ${ }^{17)}$

El profesional de enfermería debe organizar, supervisar, evaluar y promover la calidad de cuidados de enfermería, con la finalidad de brindar atención segura, oportuna, continua e intercultural de acuerdo a las políticas y normas del ministerio de salud chileno. Debe participar en los lineamientos estratégicos relacionados con la dotación de recursos humanos, materiales, físicos y financieros del cuidado de enfermería, incluyendo la administración presupuestaria asignada y la implementación de nuevas herramientas tecnológicas que faciliten el control de la gestión. Debe promover los principios éticos y legales que guían el ejercicio profesional, tanto en su rol independiente como parte de un equipo multidisciplinario, en donde se promueva la comunicación eficaz entre las diferentes dependencias institucionales. La enfermera/o debe promover un liderazgo efectivo en la gestión de los equipos de trabajo de su dependencia, para ello debe proponer programas de evaluación y mejoramiento continuo del cuidado de enfermería. Se espera también que exista una integración docente asistencial para así incentivar la investigación en el ámbito de la gestión del cuidado ${ }^{(2)}$

La enfermera cumple su función social a través de la ejecución de la gestión del cuidado, por lo que la profesión de enfermería resuelve criteriosamente un problema social determinado, como no lo podría hacer ninguna otra profesión. ${ }^{(18)}$ El criterio profesional se entiende como aquella aptitud para apreciar una situación o problema, que permite ubicarla dentro de la disciplina de enfermería y encontrar solución conforme a los principios éticos que orientan la profesión, las normas jurídicas que la regulan, los conocimientos científicos y humanistas que la avalan, los recursos de que dispone y las necesidades concretas del paciente que demanda cuidado ${ }^{(17)}$. El cuidado de enfermería corresponde a un conjunto de acciones sistematizadas, organizadas, medibles, documentables y evaluables, basadas en modelos y teorías de enfermería y/o en la evidencia científica, realizadas con el fin de garantizar que una persona, con problemas reales o potenciales de salud, reciba una atención de enfermería segura, eficiente y eficaz, cuya finalidad sea lograr su máximo bienestar ${ }^{(18)}$, esto incluye respetar y velar por los derechos del usuario en cuanto a confidencialidad, a la información en salud, a compañía, ayuda espiritual y a participar del sistema de salud chileno. ${ }^{(19)}$ 
Ceballos $^{(20)}$, plantea que además se debe trabajar en la formación de futuras/os enfermeras/os, para que éstos comprendan la importancia de los cuidados humanizados para el paciente, y la mejora de la satisfacción del usuario interno como externo.

Se debe desarrollar la capacidad de comunicación y escucha, respeto por las creencias del otro, gran dimensión de entrega, la responsabilidad y el compromiso ético en los alumnos, así como el interés de trabajar con calidad y de buscar la mejor respuesta al problema a través de las evidencias disponibles. ${ }^{(21)}$ Los docentes deben ser modelos de formación para sus alumnos, por lo que primeramente son ellos los que deben cultivar estas competencias. ${ }^{(22)}$

Resultados preliminares de un estudio efectuado por las autoras del presente trabajo, en relación al conocimiento que tenían las enfermeras académicas y asistenciales sobre la enfermería basada en la evidencia, se encontró, en ambos grupos, un bajo nivel de conocimientos sobre EBE, poca lectura crítica de los artículos científicos publicados en revistas y ninguna implementación de los resultados de las diversas investigaciones ya sea en la clínica o en la docencia. Esto deja de manifiesto que las enfermeras encuestadas en general, carecerían de la competencia necesaria para poder sustentar científicamente su quehacer y servir de modelo a las futuras generaciones. En general, manifestaron que se debía a la falta de tiempo, especialmente en la atención asistencial, resultados que también se encontraron en otros estudios. ${ }^{(14-16)}$

Para las enfermeras clínicas, EBE resulta ser una herramienta fundamental, pues permite una mejoría en la calidad de atención, basada en evidencias científicas fundamentadas en investigaciones previas, como se ha demostrado en estudios empíricos ${ }^{(23-25)}$ mejorando la gestión del cuidado, ya que se promueven cuidados oportunos y eficientes, disminuyendo las posibilidades de cometer errores asociados a la práctica. El no trabajar con evidencias se traduce en prestar cuidados erróneos, con falta de criterio y alta probabilidad de equivocación, ${ }^{(13)}$ es por ello que se debe incentivar la práctica de cuidado con evidencias que sustenten el trabajo diario de la enfermera/o, para evitar que estos sucesos ocurran, y sea el usuario quien sufra las consecuencias de la falta de responsabilidad y compromiso de las enfermeras frente a su cuidado. Además es la investigación quien hace la diferencia entre hacer y saber de las enfermeras y es por ello que se debe incentivar la práctica de enfermería con evidencias y demostrar su utilidad dentro de la profesión.

En muchas currículas de pregrado en las carreras de enfermería, no se enseña la EBE como estrategia de aprendizaje clínico ${ }^{(26)}$. La formación profesional, desde pregrado, debe motivar a la evaluación permanente con la lectura crítica ${ }^{(10)}$, el manejo de la información en la búsqueda de bases de datos, al trabajo en equipo y al manejo de una segunda lengua; para tener mayor preparación teórica y académica que permita a las nuevas generaciones enfrentarse con una mejor preparación científica. ${ }^{(15 ; 27)}$

Para poder vencer las normales resistencias al cambio y llegar a la consolidación de la nueva práctica enfermera, es preciso implementar la evidencia en la ejecución de las actividades diarias de la enfermería, con garantías de estar realizando una mejora en los cuidados. ${ }^{(28)}$

Jofré ${ }^{(29)}$ plantea que la gestión del cuidado bajo el amparo del Código Sanitario y la reforma en salud "requiere desarrollar investigación cuyos resultados se traduzcan en evidencias científicas, así como de una estructura del sistema de salud que favorezca la práctica de enfermería hacia el cuidado y se produzcan las transformaciones que muestren un quehacer profesional y humanizado". Las interrogantes que surgen en este proceso deben ser 
resueltas mediante la investigación, para generar y/o contrastar conocimientos destinados a contribuir a mejorar el cuidado en salud, lo que distingue al saber y al hacer de enfermería, lo que a su vez confiere autonomía profesional para diagnosticar necesidades de cuidado y tomar decisiones con responsabilidad ética y legal.

Para trabajar con evidencias científicas, se debe incentivar la investigación en enfermería desde pregrado con la finalidad de estimular en los alumnos la resolución de problemas asociados al cuidado de enfermería, ${ }^{\left({ }^{30}\right)}$ de tal manera que los futuros profesionales sean capaces de prestar el mejor cuidado profesional hacia el usuario, en los distintos niveles de atención del país.

\section{CONCLUSIÓN}

Sin duda la gestión del cuidado plantea desafíos de tipos administrativos y asistenciales para ser ejercidas por el profesional de enfermería que requieren de competencias y conocimientos necesarios para enfrentar lo que la sociedad espera de ellas/os. Es así que la estrategia propuesta por la EBE permite sustentar una gestión del cuidado de calidad segura y con satisfacción hacia el usuario interno como externo tomando las mejores decisiones a través de las evidencias disponibles, haciendo que las enfermeras/os sean respetados como profesionales con conductas responsables de cuidado frente a la sociedad.

\section{REFERENCIAS BIBLIOGRÁFICAS}

(1) Código Sanitario. D.F.L 725/67, 725/67, Ministerio de Salud de Chile, (1968).

(2) Subsecretaria de Redes Asistenciales, Norma General Administrativa n 19, "Gestión del Cuidado de Enfermería para la atención Cerrada", Exenta 1127, 2007.

(3) Orellana A, Paravic T. Enfermería Basada en Evidencia.Barreras y Estrategias para su Implementación. Rev. Ciencia y Enfermeria 2007;13(1):17-24.

(4) Alonso P, Ezquerro O, Fargues IGJ, Marzo M, Navarra M, Subirana M, et al. Enfermería Basada en la Evidencia. Hacia la excelencia en los cuidados. DAE ed. Madrid,España. 2004.

(5) Limón E. Investigación en enfermería. La enfermería basada en la evidencia. Rev.Investigación En Enfermería 1999;(7):35-8.

(6) Méndez M, Muñoz M, Sevilla L. Enfermería basada en la evidencia. Boletín de Enfermería de Atención Primaria 2007;4(3).

(7) Santos C, Pimenta C, Nobre M. The PICO strategy for the research question construction and evidence search. Rev Latino-Am Enfermagem 2007;15(3):508-11.

(8) Barderas A, Escobar G. Fuentes de información en Enfermería:buscando la evidencia. Rev. Enfermería Anestesia- Reanimación y Terapia del Dolor 2004;(12):447.

(9) Galvào C, Sawada N, Mendes I. A busca das melhores evidências. Rev. Esc Enfermagem 2003;37(4):43-50. 
(10) Marqués S. La Lectura Crítica. Fundamental en Enfermería y para las enfermeras. Evidentia 2009;6(27). Accesada 28 de Julio de 2010 en http/www.indexf.com/evidentia/n27/ev2779.php

(11) Sousa V, Driessnack M, Mendes I. An overview of research designs relevant to nursing: Part 1: Quantitative research designs. Rev Latino-am Enfermagem 2007;15(3):502-7.

(12) Gónzalez M. Interioriza la EBE; asumir la practica basada en evidencia. Evidentia 2005;2(4). Accesada 11 de agosto de 2010 en http/www.indexf.com/evidentia/n4/101artículo.php

(13) Gálvez A. Variaciones en la práctica médica: proyecto VPM-IRYSS. Evidentia 2005;2(6). Accesada 11 de agosto de 2010 en http/www.indexf.com/evidentia/n6/156artículo.php

(14) Retsas A. Barriers to using research evidence in nursing practice. Journal Advance Nursing 2000;31(3):599-606.

(15) Ochoa S. A propósito de la enfermería basada en la evidencia: algunos cuestionamientos, limitaciones y recomendaciones para su implementación. Rev. Invest Educ Enferm 2005;23(2):138-46.

(16) Yagüe J. Enfermería basada en la evidencia: una visión desde la práctica clínica. Evidentia 2008;5(22). Accesada 11 de agosto de 2010 en http/www.indexf.com/evidentia/n22/e6802.php

(17) Milos P, Borquez B, Larraín A. "La Gestión del Cuidado en la Legislación Chilena: interpretación y Análisis". Rev. Ciencia y Enfermería 2010;16(1):17-29.

(18) Milos P, Larrain A, Simonetti M. Categorización de Servicios de Enfermería.Propuesta para asegurar una atención de calidad en tiempos de escasez de enfermeras. Rev. Ciencia y Enfermería 2009;15(1):17-24.

(19) Mirada en Profundidad. Derechos y deberes de los pacientes: habrá mayor claridad. El Mercurio . 2007. Ref Type: Magazine Article

(20) Ceballos P. Desde los ámbitos de enfermería, analizando el cuidado humanizado. Rev.Ciencia y Enfermería 2010;16(1):31-5.

(21) Milos $P$, Larrain A, Simonetti M. Del porqué de la formación exclusiva universitaria de las enfermeras. 2009. Accesada 30 junio 2010 en http/www.colegiodeenfermeras.cl

(22) Davies B, Hughes A. Clarification of advanced nursing practice: characteristics and competencies. Rev. Clinical Nurse Specialist 2002;16(3):147-52.

(23) Borges, E. Caliri,M. Haas V. Systematic review of topic treatment for venous ulcers. Rev Latino-am Enfermagem 2007;15(6): 1163-1170

(24) Candela M. Permeabilización de catéteres venosos periféricos y centrales. La EBE no es MBE . Ciber Revista 200421. Accesada 5 de agosto de 2010 en http://ciberrevista.enferurg.com/ 
(25) Galindo S. Cómo se acerca América Latina a la Enfermería Basada en Evidencias. Evidentia 2010;7(29). Accesada 28 de julio de 2010 en http/www.indexf.com/evidentia/n29/ev0029.php

(26) Passalenti M. La gran dificultad: aplicar las evidencias a los cuidados cotidianos. Evidentia 2006;3(7). Accesada 28 de julio de 2010 en http/www.indexf.com/evidentia/n7/195artículo.php

(27) Lora-López P. Reflexiones sobre el grado y postgrado de Enfermería: la Investigación en Enfermería. Rev. Index Enferm 2010;17(2):85-6.

(28) Lacasaña P. ¿Y después de la investigación qué? Reflexión sobre la implementación de la evidencia en la práctica clínica enfermera. Evidentia 2006;3(10). Accesada 11 de agosto de 2010 en http/www.index-f.com/evidentia/n10/236artículo.php

(29) Jofré V. La investigación como herramienta de la gestión del cuidado. Rev.Ciencia y Enfermería 2009;15(3):7.

(30) Carrascosa M, García P, Bellido J, Guevara J, Morcillo M. Tendiendo puentes entre la evidencia y la práctica: estrategias de difusión para mejorar el impacto de la evidencia en la práctica enfermera . Evidentia 2004;1(1). Accesada 11 de agosto de 2010 en http/www.index-f.com/evidentia/n1/11artículo.php 